\title{
On the application of a new version of lifting surface theory to non- slender and kinked wings ${ }^{\star}$
}

\author{
TH. E. LABRUJERE \\ National Aeronautical Research Institute (N.L.R.), Amsterdam, the Netherlands \\ P. J. ZANDBERGEN \\ Department of Applied Mathematics, Twente University of Technology, Enschede, the Netherlands
}

(Received April 13, 1972)

\begin{abstract}
SUMMARY
Results are presented which are obtained by a new elaborate method developed at NLR for the determination of the characteristics of thin wings in subsonic flow. Attention is paid to the rate of convergence of the numerical solutions, especially with respect to the number of collocation points. Two rectangular wings have been treated in order to examine the influence of the aspect ratio. The influence of the rounding of a kink is demonstrated by means of a series of constant chord wings with hyperbolic edges.
\end{abstract}

\section{Introduction}

For predicting the lift distribution on wings in the subsonic speed region the lifting surface theory of Multhopp [1] has received wide acceptance. Especially the computerisation of the method by Van Spiegel and Wouters [2] has made it to the standard technique for solving aerodynamic loading problems. In essence the method is a collocation technique, applying the boundary condition at a number of pivotal points distributed over the wing in spanwise and chordwise direction, which leads to a system of linear algebraic equations.

It should be expected that the results obtained by increasing the number of spanwise and/or chordwise stations will show a certain convergence. However, it became apparent that for a given number of spanwise stations and for different numbers of chordwise points very strong variations occur in the results, becoming worse when increasing either aspect ratio or sweep of the wing.

This then led the present authors and Wouters to a thorough investigation of lifting surface theory trying to remove the inherent difficulties in Multhopp's method. Two reasons were found which may impair Multhopp's method. In the first place the spanwise integrand is not completely regular for all values of the spanwise co-ordinate. In the second place since the integrand, consisting of the product of the pressure series coefficient and the spanwise influence function is represented by a single polynomial, it is not possible to judge afterwards, whether the representation of the pressure series coefficients or the accuracy of the integral gives rise to poor results.

In [3] a new method was presented, where these difficulties were no longer present. For a number of planforms it was shown that convergence of the results occurred with an increase in the number of pivotal points. This especially proved to be true for the local center of pressure line. Although the material presented is rather convincing, two effects have not been covered, namely the effect of an increase in the number of chordwise points to about ten and the effect of rounding the planform near a kink. Especially the latter effect is important since most modern airplane wings show one or more kinks.

Therefore the purpose of the present paper is to present additional results on these items by

* This investigation has been performed under contract at the N.L.R. 
considering rectangular wings of aspect ratio two and seven and the so-called hyperbolic wing for different rounding parameters.

To make the paper self-contained a short outline is given of the more essential points in the method of [3]. Shortly after the appearance of [3] a paper was published by Lamar [5] containing the results for several planforms calculated by a somewhat modified Multhopp method. A comparison of the results of Lamar with those of the new method has been included.

\section{A short outline of the method}

If the $(x, y)$ plane is the plane of projection of a thin wing, $\alpha(x, y)$ being the local angle of incidence of the wing and $\Delta c_{p}(x, y)$ being the pressure distribution over the wing, the governing equation of lifting surface theory can be written as:

$$
\alpha(x, y)=\frac{1}{8 \pi} \cdot \int_{-s}^{+s} \int_{x_{l}\left(y^{1}\right)}^{x_{t}\left(y^{1}\right)} \Delta c_{p}\left(x^{1}, y^{1}\right) K\left(x, y ; x^{1}, y^{1}\right) d x^{1} d y^{1}
$$

where $s$ denotes the semi-span, $x_{l}$ and $x_{t}$ are the equations of the leading and trailing edge respectively, and where $K$ is given by

$$
K\left(x, y ; x^{1}, y^{1}\right)=\frac{-1}{\left(y-y^{1}\right)^{2}}\left[1+\frac{x-x^{1}}{\left\{\left(x-x^{1}\right)^{2}+\beta^{2}\left(y-y^{1}\right)^{2}\right\}^{\frac{1}{2}}}\right] .
$$

For solving the singular integral equation (2.1) $\Delta c_{p}$ is represented by

$$
\Delta c_{p}\left(x^{1}, \eta^{1}\right)=\frac{4 s}{l\left(\eta^{1}\right)} \sum_{r=0}^{R} a_{r}\left(\eta^{1}\right) h_{r}\left(x^{1}\right)
$$

where $l(\eta)$ is the local chord and $\eta=y / s$ and $X=\left\{x-x_{l}(\eta)\right\} / l(\eta)$ and where the functions $h_{r}$ depend on the Chebyshev polynomials $T_{r}$ of order $r$ as follows:

$$
h_{r}(X)=\frac{1}{\pi} \frac{T_{r}(1-2 X)+T_{r+1}(1-2 X)}{\{X(1-X)\}^{\frac{1}{2}}} .
$$

Substituting eq. (2.3) into eq. (2.1) there is obtained

$$
\alpha(X, \eta)=-\frac{1}{2 \pi} \sum_{r=0}^{R} f_{-1}^{+1} \frac{a_{r}\left(\eta^{1}\right) H_{r}\left(X, \eta, \eta^{1}\right)}{\left(\eta-\eta^{1}\right)^{2}} d \eta^{1} .
$$

The bar through the integral sign denotes the principal value, while $H_{r}$ is given by

$$
H_{r}\left(X, \eta ; \eta^{1}\right)=\int_{0}^{1} h_{r}\left(X^{1}\right)\left[1+\frac{X-X^{1}}{\left\{\left(X-X^{1}\right)^{2}+\frac{\beta^{2} s^{2}}{l\left(\eta^{1}\right)^{2}}\left(\eta-\eta^{1}\right)^{2}\right\}^{\frac{1}{2}}}\right] d X^{1}
$$

To deal with the spanwise singularities in the integrand of eq. (2.5), a regularized function $\bar{H}_{r}$ will be introduced

$$
\begin{aligned}
& \bar{H}_{r}\left(X, \eta ; \theta^{\prime}\right)= \\
& =\frac{H_{r}\left(X, \eta ; \theta^{\prime}\right)-H_{r}(X, \eta ; \theta)-\left(\cos \theta-\cos \theta^{\prime}\right)\left(\frac{\partial H_{r}}{\partial \eta^{\prime}}\right)_{\theta^{\prime}=\theta}+\frac{\beta^{2} s^{2}}{l(\eta)^{2}}\left(\cos \theta-\cos \theta^{\prime}\right)^{2} \ln \left|\cos \theta-\cos \theta^{\prime}\right|\left(\frac{d h_{r}}{d X^{\prime}}\right)_{\theta^{\prime}=\theta}}{\sin \theta^{\prime}}
\end{aligned}
$$

where $\eta=-\cos \theta$.

Now by representing the functions $a_{r}$ as well as the functions $\bar{H}_{r}$ by trigonometric interpolation formulae

$$
a_{r}\left(\eta^{\prime}\right)=\frac{2}{m+1} \sum_{n=1}^{m} a_{r}\left(\eta_{n}\right) \sum_{n=1}^{m} \sin \mu \theta^{\prime} \sin \mu \theta_{n}
$$


where

$$
\bar{H}_{r}\left(\eta^{\prime}\right)=\frac{2}{a(m+1)} \sum_{\lambda=1}^{a(m+1)-1} a_{r}(\eta \lambda) \sum_{\omega=1}^{a(m+1)-1} \sin \omega \theta^{\prime} \sin \omega \theta_{\lambda}
$$

$$
\theta_{n}=\frac{n \pi}{m+1}, \quad \theta_{\lambda}=\frac{\lambda \pi}{a(m+1)}
$$

the integration can be performed.

The integer $a$ can be used to improve the accuracy of the integration, independently of the representation of the pressure coefficient. The complete regularization of the integrand of eq. (2.5) together with the introduction of the independent representation of $a_{r}$ and $\bar{H}_{\mathrm{r}}$ are the essential new elements of the method.

Performing the integration the final linear system of equations is obtained as follows

$$
\begin{aligned}
\alpha\left(X_{p}, \eta_{v}\right)= & -\frac{1}{2} \sum_{r=0}^{R} \sum_{n=1}^{m} a_{r}\left(\eta_{n}\right)\left\{\sum_{\lambda=1}^{a(m+1)-1} \frac{\bar{H}_{r}\left(X_{p}, \theta_{v}, \theta_{\lambda}\right)}{a(m+1)} \gamma_{n \lambda}+H_{r}\left(X_{p}, \theta_{v}, \theta_{v}\right) \frac{\sin \theta_{n}}{\sin \theta_{v}} \varepsilon_{v n}+\right. \\
& \left.+\left(\frac{\partial H_{r}}{\partial \eta^{\prime}}\right)_{\theta=\theta_{v}} \zeta_{v n}-\frac{\beta^{2} s^{2}}{l\left(\eta_{v}\right)^{2}}\left(\frac{d h_{r}}{d X^{\prime}}\right)_{\theta=\theta_{v}} S_{v n}\right\}
\end{aligned}
$$

The quantities $\gamma_{n \lambda}, \varepsilon_{v n}, \zeta_{v n}$ and $S_{v n}$ are given in the following table

\begin{tabular}{lc}
\hline$\frac{(-1)^{n} \sin \frac{\lambda \pi}{a} \sin \theta_{n}}{(m+1)\left(\cos \theta_{n}-\cos \theta_{\lambda}\right)}$ & $\theta_{n} \neq \theta_{\lambda}$ \\
\hline$\gamma_{n \lambda} \frac{\theta_{n}=\theta_{\lambda}}{1}$ & $\theta_{v} \neq \theta_{n}$ \\
\hline$\frac{(-1)^{n} \sin (m+1) \theta_{v}}{\cos \theta_{v}-\cos \theta_{n}}+\frac{1}{m+1} \frac{1-(-1)^{n} \cos (m+1) \theta_{v}}{\left(\cos \theta_{v}-\cos \theta_{n}\right)^{2}} \sin \theta_{v}$ & $\theta_{v}=\theta_{n}$ \\
\hline$\varepsilon_{v n} \frac{\frac{m+1}{2}}{\sin \theta_{n}}$ & $\theta_{v} \neq \theta_{n}$ \\
\hline$\zeta_{v n} \frac{\sin \theta_{n} \frac{1-(-1)^{n} \cos (m+1) \theta_{v}}{\cos \theta_{v}-\cos \theta_{n}}}{0} \frac{\theta_{v}=\theta_{n}}{S_{v n}} \frac{2}{m+1}\left[\left(-\frac{1}{2} \ln 2+\frac{1}{4} \cos 2 \theta_{v}\right) \sin \theta_{n}-\sum_{\mu=2}^{m} \frac{\left(\mu \sin \mu \theta \sin \theta+\cos \mu \theta_{v} \cos \theta_{v}\right)}{\mu^{2}-1} \sin \mu \theta_{n}\right]$ & \\
\hline
\end{tabular}

For the computation of the further quantities occurring in eq. (2.9), especially $\bar{H}_{r}\left(X_{p}, \theta_{v}, \theta_{v}\right)$ the reader is referred to [3].

As will be clear the collocation points $\left(X_{p}, \eta_{v}\right)$ can be chosen arbitrarily. However, in the present analysis the usual Multhopp distribution has been used, i.e.

$$
X_{p}=\frac{1-\cos \frac{2(p+1) \pi}{2 R+3}}{2}, \quad p=0, \ldots, R
$$

and

$$
\theta_{v}=\frac{v \pi}{m+1}
$$

The method has been programmed in ALGOL. This program together with the appropriate input and output listings have been described in [4]. 
After this short outline of the method itself, the attention will now be focussed on the systematic study of wings with many chordwise pivotal points and on the description of the lift of kinked wings.

\section{Discussions of results}

Three wings have been considered, the planform of which is shown in fig. 1. In order to examine the influence of the aspect ratio, calculations have been performed for two rectangular wings with aspect ratio 2 and 7 respectively.
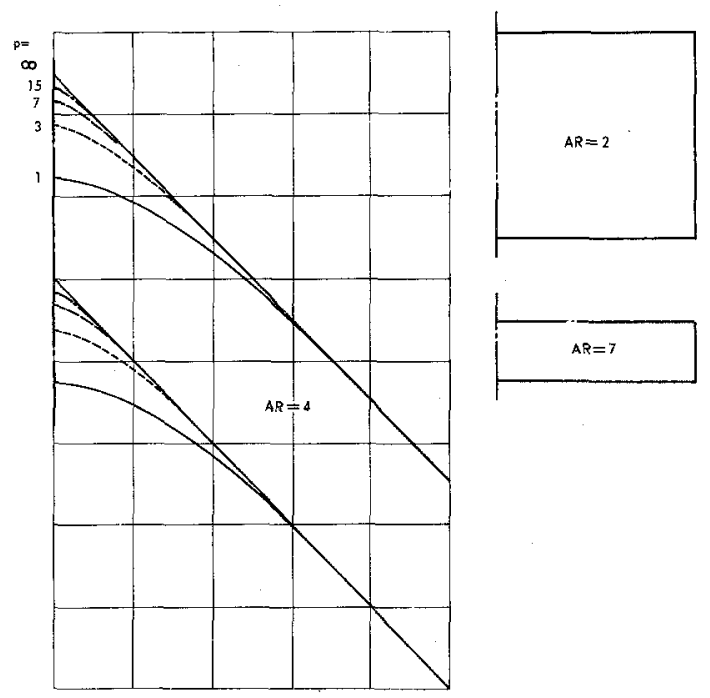

Figure 1. Wing planforms.

Here special attention is given to the number of chordwise stations to acquire a good description.

In order to examine the influence of kinks and the effect of its rounding off, calculations have been performed for a series of wings having a swept planform of constant chord, aspect ratio 4 and hyperbolic leading and trailing edges defined by:

$$
\begin{aligned}
& X l=\frac{2 p+1}{2 p(p+1)}\left\{1+p(p+1) y^{2}\right\}^{\frac{1}{2}}-\frac{1}{2 p(p+1)} \\
& X t=\frac{2 p+1}{2 p(p+1)}\left\{1+p(p+1) y^{2}\right\}^{\frac{1}{2}}+\frac{2 p^{2}+2 p-1}{2 p(p+1)} \quad 0 \leqq|y| \leqq 1
\end{aligned}
$$

where $p$ is an integer constant. By considering the values $1,3,7$ and 15 a series of wings is obtained with an increasingly severe rounding of the kink of the center section.

The results obtained by the present method for the rectangular wings have been compared with the results obtained by Lamar [5] for the same wings. Lamar has applied Multhopp's method [1] modified with respect to the method for solving the system of linear equations, the numerical evaluation of the chordal integrals and the maximal number of chordal collocation points allowed. These modifications do not affect the original scheme of Multhopp's solution to the subsonic lifting surface problem. So Lamar's results can be used to demonstrate the improvement obtained by the present method with regard to Multhopp's method.

Fig. 2 presents the results for the rectangular wing of aspect ratio $2 . d C_{l} / d \alpha$ and $X_{a c}$ are presented as a function of the number of spanwise and chordwise collocation points ( $m$ and $R$ ).

It will be clear that it is in fact impossible to give an opinion on the correct solution with the aif of Lamar's results which are presented by the solid lines, whereas the results of the present 

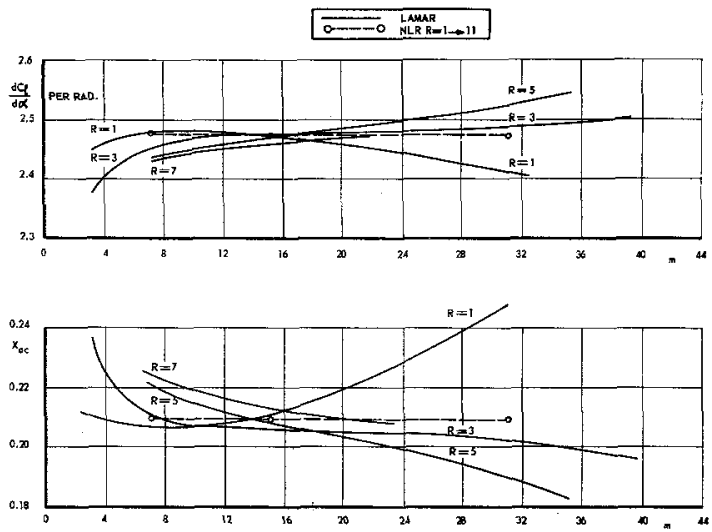

Figure 2. Lift curve slope and location of aerodynamic centre for the rectangular $(A R=2)$ as a function of the parameters $m$ and $R$.

method are identical within drawing accuracy. It will be demonstrated below by means of the results obtained by the present method, that this is due to the fact that the spanwise integrations are inaccurate.

The present method, as has already been explained, offers the possibility to apply a number of spanwise integration points different from the number of spanwise collocation points, thus achieving an improvement of the spanwise integration accuracy without increasing the number of spanwise collocation points.

The effect of this feature has been investigated by a large number of calculations, the results of which are presented in table 1 .

It is evident from the first part of this table, that by adjusting the number of spanwise integration points $(a(m+1)-1)$ an accurate result is obtained at each value of $R$. Furthermore it appears that $a$ has to be increased if $R$ is increased.

From the second and third part of table 1 it appears that convergence with respect to $R$ may be obtained when an appropriate value of $a$ is chosen.

From the last part it appears that the solution to the given problem is obtained for $m=7$, $R=7$ and $a=32$ with an accuracy of 4 to 5 significant figures.

Furthermore it can be seen that the number of spanwise collocation points is hardly significative to the solution as long as the number of spanwise integration points is sufficient.

From these considerations it follows that Lamar could in fact not hope to arrive at an equally accurate solution being restricted to orders of about 100 for the system of linear equations to be solved, whereas within this method a number of $\frac{1}{2} a(m+1)(R+1)=128 \times 8=1024$ equations would have been required ( $m=255$ ).

Finally it may be remarked that a solution to the given problem is apparently already obtained for $m=7, R=1$ and $a=4$ (32 equations in Multhopp's method) which is accurate in about 3 significant figures. It may be true that Lamar's solution for $R=1, m=7$ approximates the true solution fairly well but from fig. 2 it may be concluded that this is purely accidental.

From table 2 and figure 3 it appears that the same remarks hold for the rectangular wing with aspect ratio 7 . A solution to this problem, accurate in 4 significant figures is obtained by the present method for $m=15, R=3$ and $a=12$, whereas a solution accurate in 3 significant figures is obtained for $m=15, R=1, a=8$. Again it becomes clear that Multhopp's method would give rise to quite a large system of equations in order to arrive at a solution with the same accuracy.

From comparison of the results for these two wings it can be seen that an increase in aspect ratio gives rise to a decrease in the number of chordal collocation points required to obtain a solution of prescribed accuracy. The number of spanwise integration points on the contrary is hardly influenced by a change in aspect ratio.

Though the present method no more than Multhopp's method is suited to treat wings with 
Th. E. Labrujere, P. J. Zandbergen

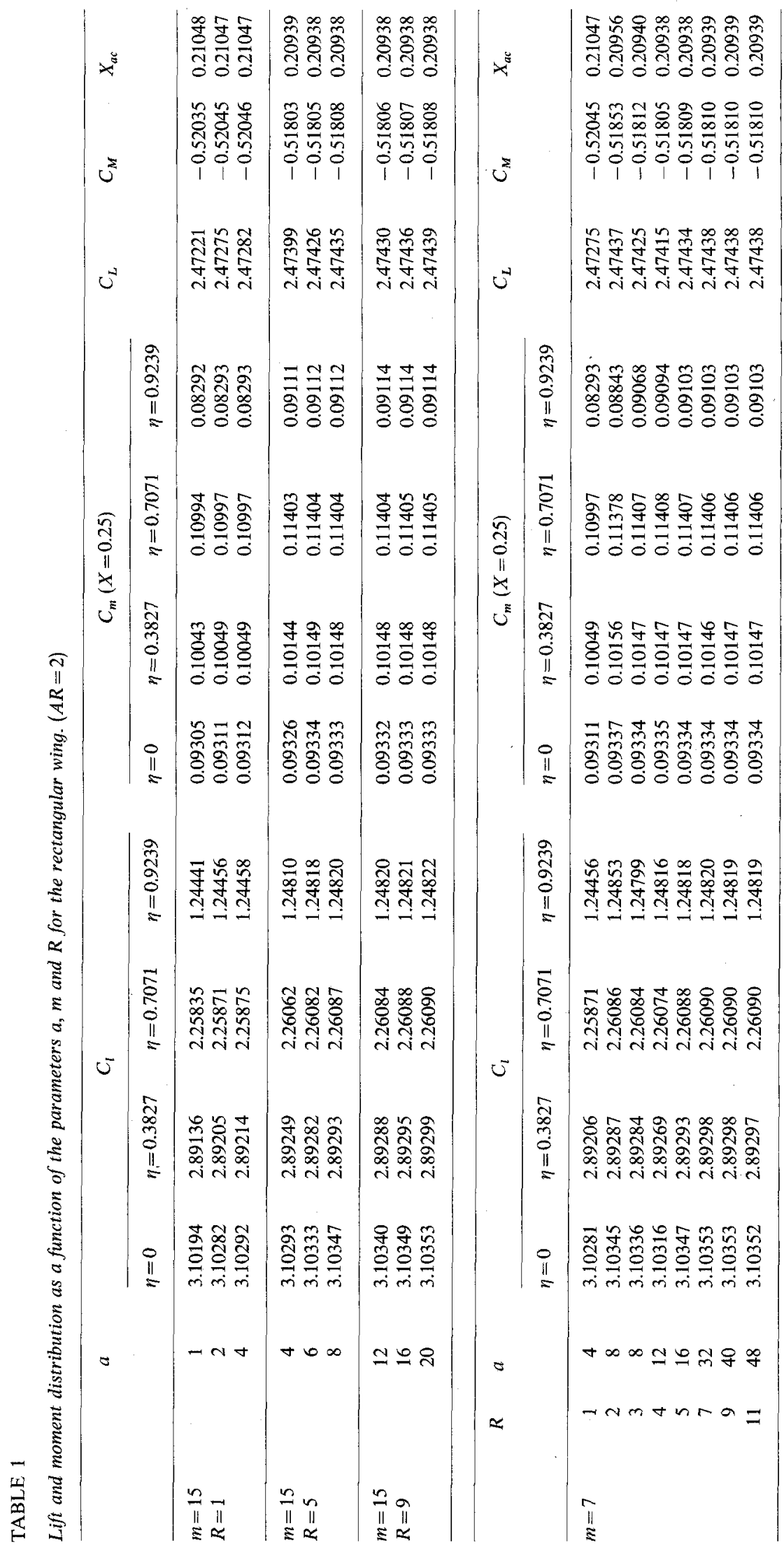




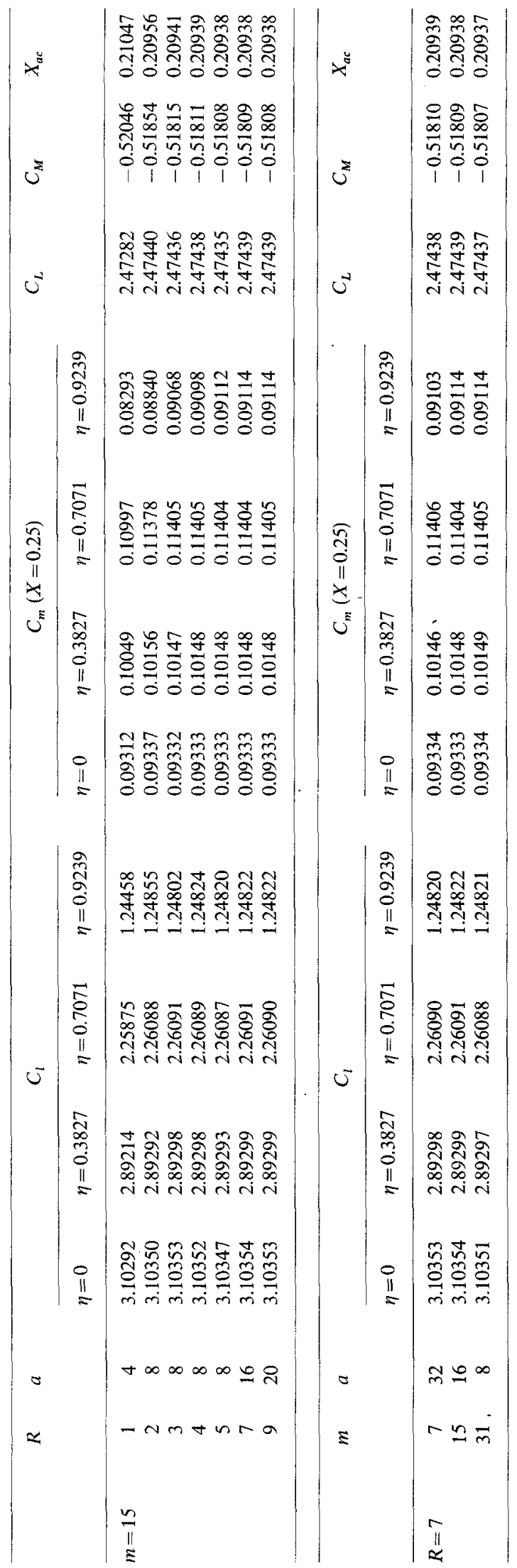


Th. E. Labrujere, P. J. Zandbergen

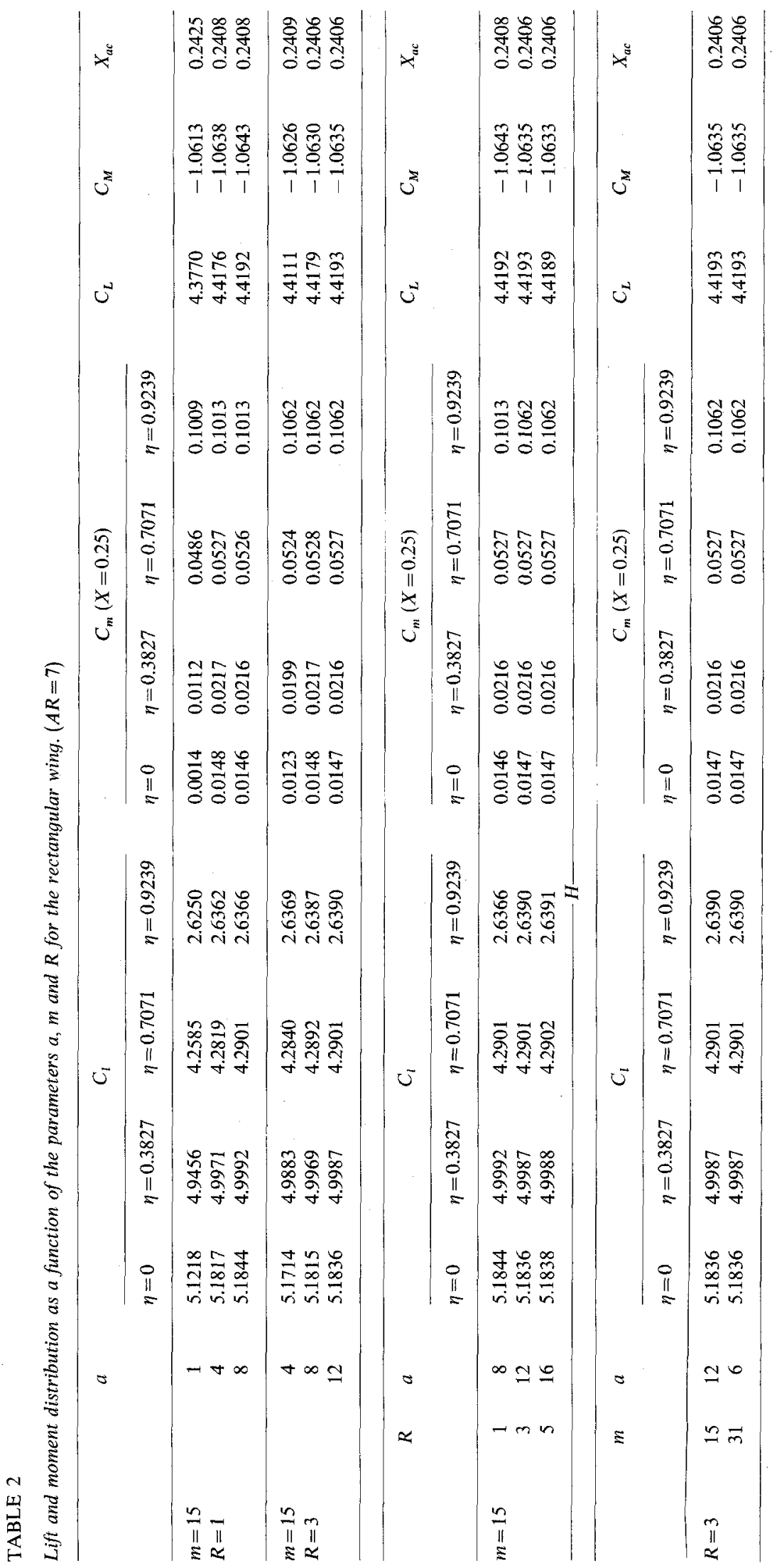



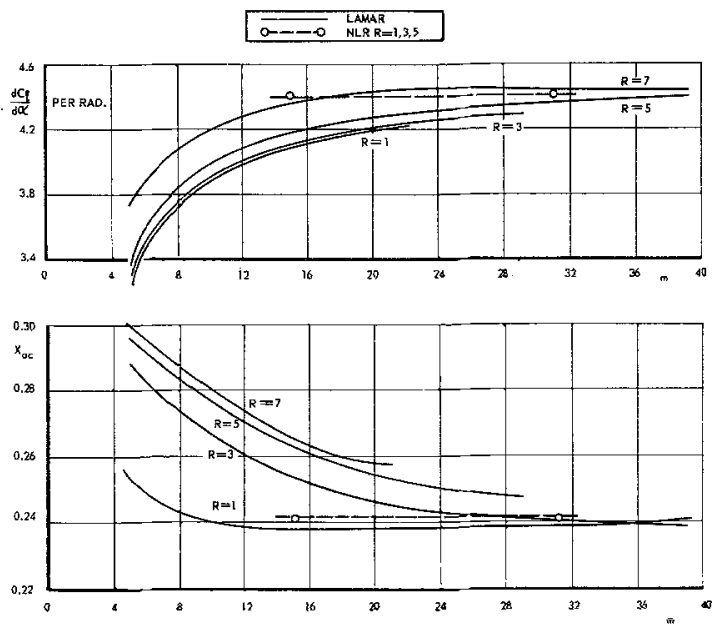

Figure 3. Lift curve slope and location of aerodynamic centre for the rectangular wing $(A R=7)$ as a function of the parameters $m$ and $R$.
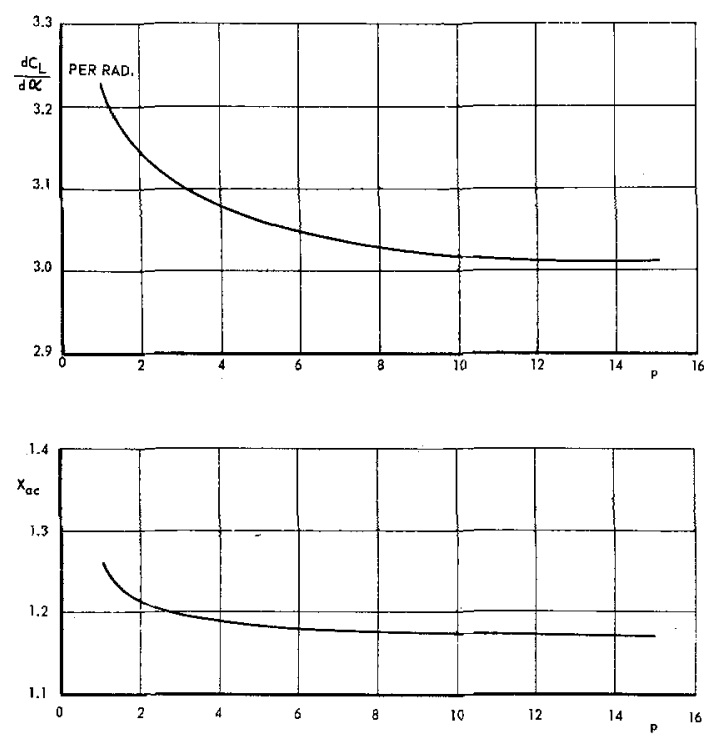

Figure 4. Lift curve slope and location of aerodynamic centre as a function of the rounding parameter $p$.

kinked planforms it may be attempted to find a solution to problems of this kind by rounding off the kinks such that the radius of curvature remains continuous. The question to be answered is then to what extend the solution on the remainder of the wing is influenced by this procedure.

In order to get an insight into this problem the hyperbolic wing planforms mentioned above have been considered. Table 3 presents a survey of the results.

It can be seen that the solution for the different roundings is rather insensitive to the number of chordal collocation points. Also the number of spanwise integration points is nearly invariant. The number of spanwise collocation points on the contrary has to be increased when more severe roundings are considered.

As for the local solution at the centre section it may be remarked that there seems to exist a very slow convergence with respect to the rounding parameter $p$.

Furthermore it can be seen that the influence of the rounding on the local solution at the outboard sections decreases as the rounding becomes more severe.

The overall values of $d C_{l} / d \alpha$ and $X_{a c}$ show a distinct convergence with respect to the rounding parameter $p$ as is illustrated by fig. 4 .

It may be concluded from these results that for $p=15$, the local lift curve slope in the centre 
Th. E. Labrujere, P. J. Zandbergen

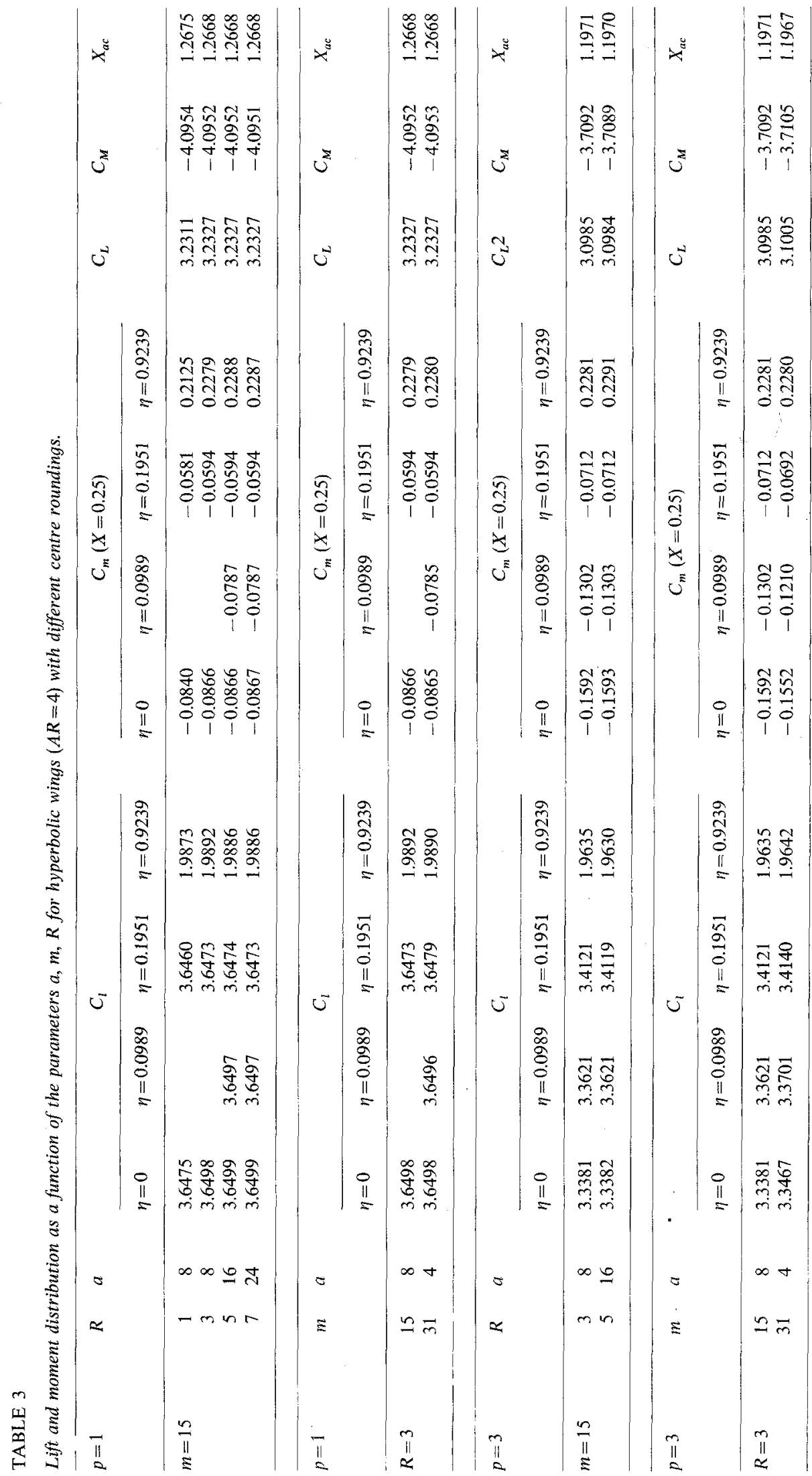




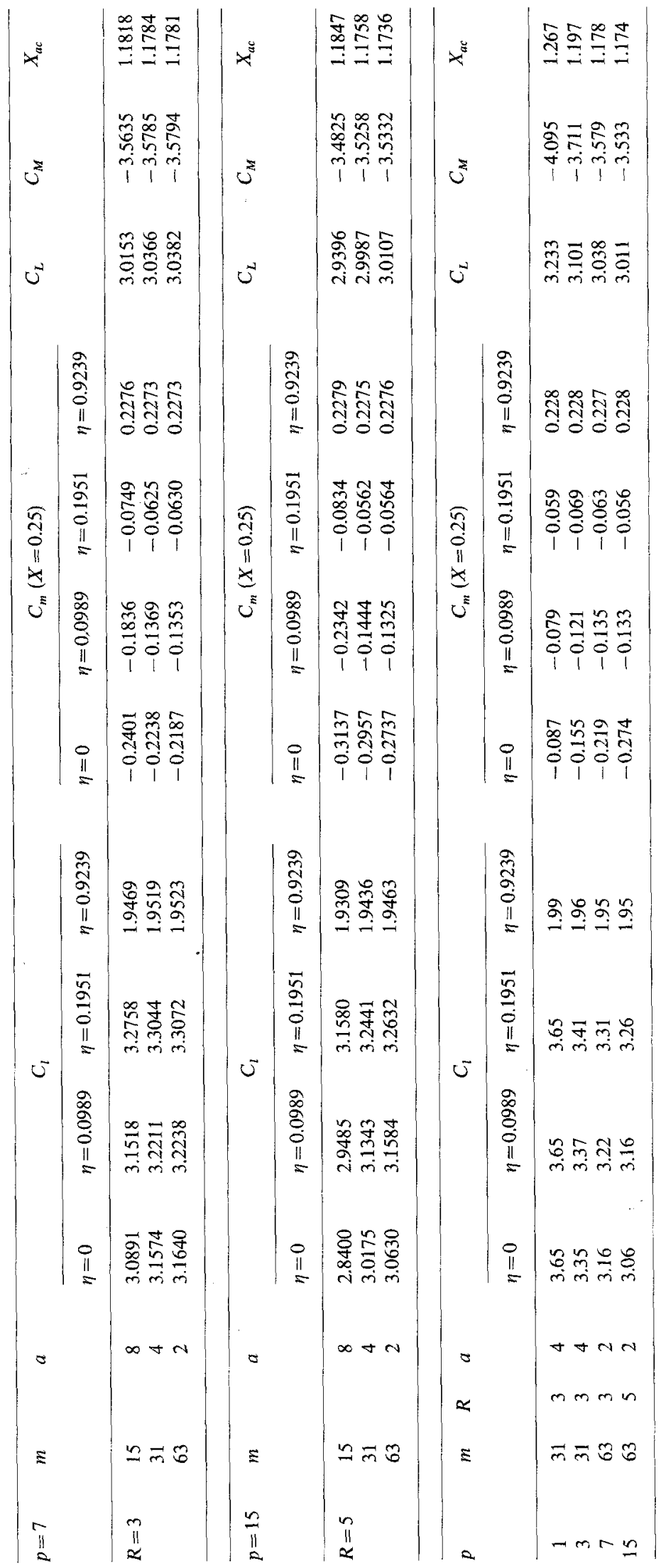


of the wing is about $3 \%$ in error, while the total quantities are less than $1 \%$ in error. This then makes it obvious that fair results can be obtained by applying a rounding $p=15$. For real accurate results, however, larger values of $p, m$ and $R$ have to be chosen, making the method prohibitive because of the enormous increase in computing time.

\section{Conclusions}

From the results presented it may be concluded that the method of [3] offers the possibility to determine very accurate solutions to the subsonic lifting-surface problem.

The method has been formulated such that exact results can be found for wings with continuous leading and trailing edge curvature only. Nevertheless it appears possible to determine fairly accurate overall characteristics for wings with kinked leading and trailing edges by applying an appropriate rounding of the kinks. The rounding has only local influence.

An increase of the aspect ratio gives rise to a decrease of the number of chordal collocation points required to obtain a given accuracy whereas the number of spanwise collocation points is rather invariant as long as the number of spanwise integration points is sufficient.

Furthermore it has been shown that the present method establishes a considerable improvement with respect to Multhopp's method especially concerning the numerical convergence.

\section{REFERENCES}

[1] H. Multhopp, Methods for calculating the lift distribution of wings (Subsonic lifting-surface theory), A.R.C. R and M $2884,(1955)$.

[2] E. van Spiegel and J. G. Wouters, Modification of Multhopp's lifting-surface theory with a view to automatic computation, NLR report TN.W.2, 1962.

[3] P. J. Zandbergen, Th. E. Labrujere and J. G. Wouters, A new approach to the numerical solution of the equation of subsonic lifting surface theory, NLR TR 649, November 1967.

[4] Th. E. Labrujere and J. G. Wouters, Computer application of subsonic lifting-surface theory, NLR TR 70088 C, September 1970. 